\title{
Feasibility of short term drainage for diagnostic thoracoscopy
}

\author{
D.P. Breen ${ }^{1}$, S. Mallawathantri1,2, A. Fraticelli1, L. Greillier1, P. Astoul1
}

ABSTRACT: Feasibility of short term drainage for diagnostic thoracoscopy. D.P. Breen, S. Mallawathantri, A. Fraticelli, L. Greillier, P. Astoul.

Background and Aim. Thoracoscopy is a diagnostic tool superior to other available techniques for the assessment of pleural effusions. There are numerous publications that describe the technique in detail but there is very little published on the optimal time of chest drain removal post procedure. Our aim was to retrospectively study all cases of diagnostic thoracoscopy and to ascertain the time of chest drain removal, length of hospital stay and associated complications.

Methods. All patients who underwent thoracoscopy during a 6-year period were identified from a computerised database. Patients who received talc for pleurodesis were excluded as they required longer drainage time. A review of the remaining patients' charts and radiology was performed to ascertain the predefined outcomes.
Results. 124 patients had a diagnostic thoracoscopy. The time to chest drain removal was documented as less than four hours, four to $\mathbf{2 4}$ hours, 24 to $\mathbf{4 8}$ hours and greater than 48 hours in $66(53.2 \%), 29(23.4 \%), 12(9.7 \%)$ and $17(13.7 \%)$ of patients respectively. The median length of stay for all patients was one day (interquartile range, 1-4 days). There was a statistically significant difference in overall length of hospital stay between the early ( $<4$ hours) and late ( $>48$ hours) chest drain removal groups, $p=0.0028$. The overall complication rate was $15.9 \%$. There was no statistical difference in complication rates between the two groups.

Conclusion. This retrospective series demonstrates that early chest drain removal post diagnostic thoracoscopy is possible and safe. This is likely to confer economic benefits.

Monaldi Arch Chest Dis 2009; 71: 2, 54-58.

Keywords: Thoracoscopy, Chest drain, Early removal.

${ }^{1}$ Faculty of Medicine (Université de la Méditerranée), Assistance Publique Hôpitaux de Marseille, Department of Pulmonary Diseases, Division of Thoracic Oncology, Hôpital Sainte-Marguerite, Marseille, France.

2 Department of Respiratory Medicine, Nottingham University Hospitals NHS trust, City Hospital Campus, Nottingham, U.K.

'David Patrick Breen is the recipient of a European Respiratory Society/ European Lung Foundation Fellowship (Number 21)'.

Correspondence: Philippe ASTOUL, Faculty of Medicine (Université de la Méditerranée), Assistance Publique Hôpitaux de Marseille, Department of Pulmonary Diseases, Division of Thoracic Oncology, Hôpital Sainte-Marguerite, 13274 Marseille Cedex09, France; e-mail: pastoul@ap-hm.fr

\section{Introduction}

Thoracoscopy was first performed in 1866 by Francis Richard Cruise. However, it was not until 1910, that the technique was described in detail by Jacoboeus when he advocated its use for the diagnosis of pleural disease of unknown aetiology and for obtaining macroscopic biopsies [1].

Today, thoracoscopy is performed by physicians (medical thoracoscopy) primarily for diagnosis and pleurodesis and by surgeons [video-assisted thoracoscopic surgery (VATS)] where it permits more complex thoracic surgical procedures [2].

There are a number of guidelines, statements and state-of-the-art publications on the use of thoracoscopy for diagnostic, therapeutic and advanced indications [3-5]. However, these guidelines do not address the issue of chest drain removal post-procedure. In the setting of a diagnostic procedure without talcage, if it is feasible to remove the drain early, it could allow early discharge of the patient and this may reduce complications related to prolonged drainage and in turn have an economic benefit to the health service by reducing the overall length of stay [6].
The aim of our study is to retrospectively assess the time of chest drain removal post-thoracoscopy and the length of in-patient stay post-procedure.

\section{Materials and methods}

All consecutive patients who underwent a medical thoracoscopy in our unit from January 2001 to December 2006 were included in the study. These patients were identified from the department's computerised database. All subjects who underwent talcage for pleurodesis were excluded from the study. We performed a review of the discharge summaries, charts and radiology of all the remaining cases. The study protocol was conducted in accordance with the local research policies. Informed consent was not required by the local ethics committee as the study only entailed a retrospective review of the patients' medical records.

Thoracoscopy was performed as previously described $[2,7]$. Briefly all patients had an anaesthetic consult prior to the planned procedure. Thoracoscopy was usually performed with the patient 
under general anaesthesia and spontaneously ventilating through a single lumen endotracheal tube. The site of incision was chosen depending upon the presumed aetiology; the second or third intercostal spaces for a pneumothorax and the fifth or sixth intercostal spaces for a pleural effusion. After inducing a pneumothorax with a pleural needle, blunt dissection was performed thus creating access to the pleural space via a trocar. A single port of entry was used in all cases. In the case of a pleural effusion, all fluid was aspirated to create a dry pleural space. A systematic examination of the cavity was performed and if indicated biopsies were performed. A chest drain was inserted under direct vision at the end of the procedure and connected to an underwater seal. A functioning drain was confirmed visually by observing bubbling in the system. Once observed, the system was connected to negative pressure ranging from -10 to $50 \mathrm{~cm} \mathrm{H}_{2} \mathrm{O}$. The negative pressure was decreased step-by-step until bubbling stopped signifying the opposition of the two layers of pleura. A chest radiograph was performed to confirm radiographically that full lung re-expansion had occurred. Therefore, in all patients the chest drain was removed after a clinical examination of the patient and chest drain and confirmation of full re-expansion radiographically. The drain was then removed and the wound was closed with a single suture, which was removed after 10 days.

\section{Outcomes}

The primary outcome of the study was the time to chest drain removal. Secondary outcomes were the length of hospital stay post thoracoscopy and complications occurring in the peri- and post-thoracoscopy period. A major complication was retrospectively defined as events requiring active medical management during the inpatient stay. Minor complications were events that required medical supervision only. The duration of drainage was measured from the time of drain insertion at the end of the thoracoscopy to the hour or day the chest drain was removed. This was obtained from the medical notes or where a specific time was not documented, from the time on the post drain radiograph. This was then subdivided into 4 groups; one to four hours, four to 24 hours, 24 to 48 hours and greater than 48 hours. In all cases, radiographs post drain removal were reviewed for evidence of a pneumothorax or subcutaneous emphysema.

\section{Statistics}

All data was analysed using graph pad prism software (Graph Pad Software Inc. San Diego, California) Quantitative values are presented as mean +/- standard deviation (SD) when normally distributed and nominal variables are presented as percentages. The median and interquartile range was used when the distribution was non-Gaussian. An unpaired $t$ test was performed to examine for significant differences between the early and late drain removal groups for length of hospital stay.

\section{Results}

There were 428 thoracoscopies performed between January 2001 and December 2006. All patients who underwent talcage for pneumothorax or pleural effusion were excluded from the analysis as they required the chest drain to remain in situ for a longer period. Therefore 124 cases were identified and this paper reports the results from these cases.

Baseline patient characteristics are shown in table 1. Thoracoscopy was performed on the right pleural cavity in 81 cases $(65.3 \%)$ and left cavity in 43 patients $(34.7 \%)$. The cavity was described as free, with mild, moderate or severe adhesions in 58 (46.8\%), 33 (26.6\%), $26(21.0 \%)$ and 7 (5.6\%) respectively. Biopsies of the parietal pleura were performed in 111 cases $(89.5 \%)$. The median number of biopsies was 15 (interquartile range, 12-20). There were no biopsies performed in 13 cases $(10.5 \%)$. The thoracoscopic appearance was described as neoplastic, non specific inflammation, normal pleura, empyema/infectious and no access (to the pleural space secondary to adhesions) in 65 (52.4\%), 39 (31.5\%), 9 (7.3\%), 6 (4.8\%) and 5 $(4.0 \%)$ patients respectively (figure 1$)$. Post procedure the majority of patients $(n=107)$ had a $28 \mathrm{f}$ drain inserted. The only exceptions to this were cases with poor or limited access when a smaller drain was utilised and in the cases of empyema when a larger drain was used to facilitate ongoing drainage and lavage.

The time to chest drain removal was documented as less than four hours, four to 24 hours, 24 to 48 hours and greater than 48 hours in $66(53.2 \%), 29$ $(23.4 \%), 12(9.7 \%)$ and $17(13.7 \%)$ of patients respectively, (figure 2). The median length of stay was

\begin{tabular}{lc} 
Table 1. - Baseline patient characteristics \\
\hline Sex n (\%) & $99(79.8 \%)$ \\
Male & $25(20.2 \%)$ \\
Female & $66.2+/-12.6(32-88)$ \\
\hline Age & \\
\hline Diagnosis & 73 \\
Neoplasm & \\
- Mesothelioma & 43 \\
- Lung Primary & 6 \\
- Breast cancer & \\
- Adenocarcinoma & 3 \\
- Poorly differentiated cancer & \\
- Lymphoma & 18 \\
Non Specific Pachypleuritis & 33 \\
Empyema secondary to pneumonia & 12 \\
T.B. & 4 \\
Non-malignant effusion in Breast & \\
Cancer & 1 \\
Yellow nail syndrome & 1 \\
\hline
\end{tabular}




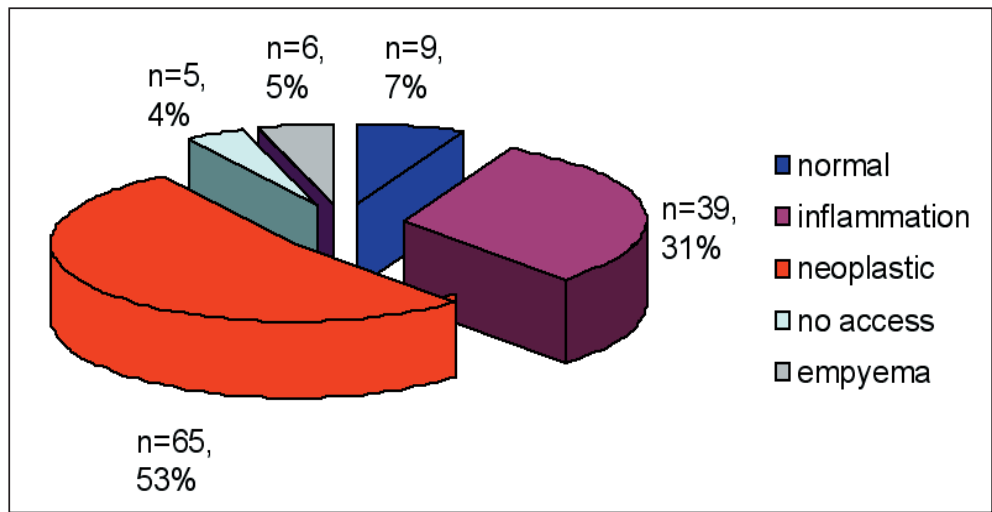

Fig. 1. - Figure 1 demonstrates the gross appearance of the pleura in the 124 patients as described by the performing physician at the time of thoracoscopy. n, number; $\%$, percentage.
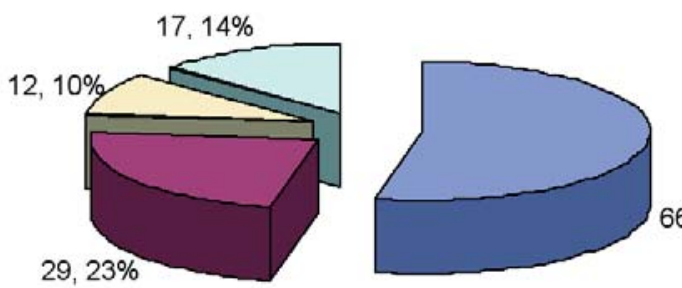

\section{$\square<4 \mathrm{hrs}$ प4-24hrs व24-48hrs a $>48 \mathrm{hrs}$}

Fig. 2. - Time to chest drain removal in the cohort of 124 patients. n, number; $\%$, percentage.

one day (interquartile range, 1-4 days). When the two subgroups of less than 4 hours and greater than 48 hours were compared, there was a statistically significant difference in overall length of hospital stay between the groups, ( $<4$ hours group; median LOS 1 day, minimum 1 day, maximum 27 days; $>48$ hours group; median LOS 7 days, minimum 3 days, maximum 34 days; $p=0.0028$ ).

There were a total of 19 complications for this cohort, equating to a complication rate of $15.9 \%$. This could be subdivided into 10 major and $9 \mathrm{mi}-$ nor complications (table 2 , figure 3 ). In each of the

Table 2. - Major and minor complications of thoracoscopy

\begin{tabular}{ll}
\hline Major: (n, \%) & $10(8.1 \%)$ \\
Pneumothorax (aspiration) & 1 \\
Pain & 2 \\
Pyrexia & 2 \\
Delirum & 1 \\
Acute abdomen & 1 \\
Decortication for empyema & 2 \\
Peripheral oedema & 1 \\
\hline Minor: (n, \%) & $9(7.2 \%)$ \\
Pneumothorax (observation) & 6 \\
Subcutaneous emphysema & 1 \\
Prolonged bubbling & 2 \\
\hline Death: (n, \%) & $2(1.7 \%)$ \\
\hline
\end{tabular}

four to 24 hours, 24 to 48 hours and greater than 48 hours complications occurred in eight, three, one and seven patients respectively. In the latter two groups, seven of the eight documented complications were related to the pleural space; prolonged bubbling and persistent pneumothorax. In the former two groups, complications were related to non pleural events in six cases, (pain $(n=2)$; pyrexia $(n=2)$; delirium $(n=1)$; leg oedema $(n=1))$ and pleural associated in five cases, (pneumothorax not requiring intervention $(n=3)$; decortication for tubercular empyema $(n=1)$ and subcutaneous empyema $(n=1))$. There were two deaths within the cohort. Neither death was directly related to the procedure but as a result of the underlying disease state, (broncho-pleural and pleuro-oesophageal fistulae in two patients with non small cell lung cancer and oesophageal cancer respectively).

\section{Discussion}

The role of thoracoscopy as a diagnostic and therapeutic tool for talcage is undisputed [8]. However there is very little published about post thoracoscopy care and, in particular, the time of removal of the chest drain post procedure, particularly in the setting of a diagnostic thoracoscopy. Both guidelines and reference texts are vague on this subject $[3,4,9]$. The reference textbook entitled "Practical Thoracoscopy" by Boutin et al advises that the drain "may be quickly removed as soon as the effectiveness of the manoeuvre is confirmed radiographically" [7]. However in Interventional Pulmonology guidelines and state of the art publications it is advised to insert a chest drain but no specific timing is documented for removal

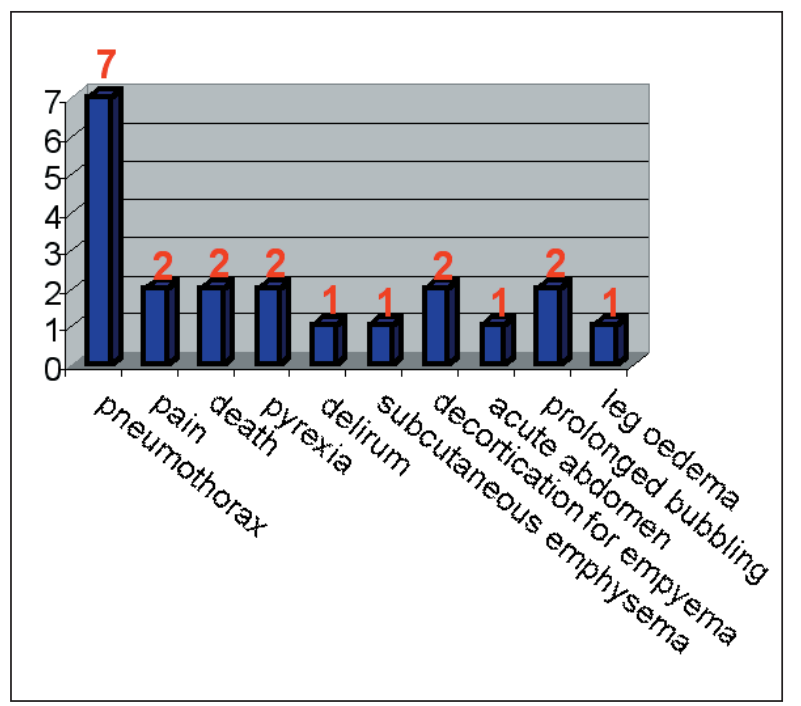

Fig. 3. - Figure 3 lists all complications occurring in the post thoracoscopy period. 
of the drain $[3-5,10]$. Within the VATS literature, there is one paper which randomised patients to a chest drain or post VATS. This paper demonstrated that it was possible to perform a VATS procedure without inserting a chest drain if there was no air leak demonstrated by a water test. The authors demonstrated that the group without a drain had a decreased rate of pneumothorax and a shorter hospital stay [11].

In this paper, we retrospectively investigated our experience with post thoracoscopy care and in particular the timing of drain removal and its effect on hospital length of stay.

In $76 \%$ of cases the drain was removed within 24 hours and in 53\% of cases within the first four hours post procedure. The median length of hospital stay for all patients was one day. However patients with early chest drain removal had a statistically significant earlier discharge compared to those who required prolonged drainage. The overall complication rate in this series was $15.9 \%$, which is comparable to the complication rates documented in the literature [12]. There was no significant difference in complication rates between patients who had the chest drain removed within 24 hours and those who had the drain in situ for a longer period. In patients with prolonged drainage, complications were predominantly related to persistent pleural sequelae that necessitated the drain remaining in situ for a longer period. The pneumothoraces in the early drain removal groups were related to the drain removal. However, no patient required reinsertion of the tube.

Although all of these procedures were defined as diagnostic, approximately $10 \%$ of patients did not have biopsies performed. This was because no access to the pleural space was possible secondary to adhesions $(n=5)$ or the thoracoscopy was consistent with emphysema and loculations $(n=8)$.

Although these thoracoscopies performed on intubated patients under general anaesthesia we do believe that the procedures are distinct from surgical VATS. In all our cases the thoracoscopies are performed in the endoscopy unit and only one port of entry is employed. This differs from VATS performed in the operating theatre where three ports of access are frequently used and more complex procedures can be performed.

This retrospective study demonstrates that it is feasible and safe to remove the chest drain within the first 24 hours and that this in turn has an effect on the overall length of hospital stay. This has obvious health economic benefits. The shortage of hospital beds and the increasing pressure on hospital budgets means that there is a drive to shorten the overall hospital stay. This paper demonstrates that diagnostic thoracoscopy, which is the most accurate technique to obtain a specific diagnosis can be performed during a short hospitalisation and thus fulfils the requirements of modern health economics.

In all cases radiographs were performed pre and post chest drain removal. However, this may be overcautious. It is possible that a careful clinical examination pre drain removal may be suffi- cient. In addition, a post drain removal radiograph may be unnecessary unless there are specific symptoms. In this series there were seven pneumothoraces but only one patient was symptomatic and required specific treatment. In bronchoscopy literature, Izbicki et al performed a prospective study to investigate if a radiograph post transbronchial biopsy is necessary [13]. They concluded that an x-ray was only necessary if the patient was symptomatic. When a pneumothorax was observed it was usually small and asymptomatic. It is likely that the same approach could be advocated for post thoracoscopy care. However, this needs to be confirmed in prospective studies. Pleural ultrasound may be another option to outrule a pneumothorax prior to drain removal. Characteristic ultrasonographic features are associated with a pneumothorax. Ultrasound has a diagnostic effectiveness superior to chest radiography in diagnosing occult pneumothorax post intervention [14-16]. However, the accuracy of ultrasound in assessing the presence of a pneumothorax may decrease over time particularly if a tube thoracostomy is inserted [17]. As far as we are aware, no study has been performed to assess the role of ultrasound in the post thoracoscopy period.

A number of caveats must be highlighted. This is a retrospective study with associated inherent faults. These post drain removal x-rays are performed at variable times. Therefore the length of drainage is not accurate in all cases. However, this method overestimates the length of drainage and therefore it is likely that more patients had their drains removed within the first 4 hours. The use of bedside pleural ultrasound may overcome this problem. In addition, it was not possible to perform an economic assessment of this management approach. This assessment would be very desirable and will be performed during future prospective studies. Finally, it was frequently unclear in this retrospective review why drains were removed at a particular time. This may be related to physician's experience or preference or simply to the timing of the post procedure assessment. However, this was not the primary objective of this paper. Our aim was to demonstrate that early removal of the drain was feasible and safe. In $76 \%$ of cases this was possible within 24 hours without any increase risk of complications.

Initially, we wanted to demonstrate that it is possible to remove the drain "on the table". This is the preferred practice of our unit and in our experience it is associated with short length of stay and less pain [6]. Overall complication rates of drainage range from $9.1 \%$ to $30 \%$ with the rate of empysema varying from $1 \%$ to $8 \%$ [18]. Early drain removal decreases these complication rates. In addition, Baumann presented a comprehensive review of the literature in Current Opinion in Pulmonary Medicine where he discusses drain size, duration of drainage and related management issues. He concludes that there is a need for more prospective studies in this field [19]. However, most of these management issues are of minimal importance if the drain can be removed early in the 
setting of a diagnostic thoracoscopy. It also has the advantage that patients can return to the ward without a drain and this reduces the pressures on the nursing staff as well as negating the need for ongoing in-service education to provide the best care for patients with chest drains $[20,21]$. However, in a retrospective review it was frequently impossible to confirm precisely if the drain was removed in the endoscopy suite. This needs to be documented in a prospective study which should also look at pain scores, length of stay and a comprehensive economic assessment of this approach.

In conclusion, taking into account the diagnostic efficacy of thoracoscopy for pleural diseases [22], we have demonstrated in this retrospective series that early chest drain removal post diagnostic thoracoscopy is possible and safe with likely economic benefits. Further prospective studies are needed in this area.

\section{References}

1. Moisiuc FV, Colt HG. Thoracoscopy: origins revisited. Respiration 2007; 74: 344-55.

2. Rodriguez-Panadero F, Janssen JP, Astoul P. Thoracoscopy: general overview and place in the diagnosis and management of pleural effusion. Eur Respir J 2006; 28: 409-22.

3. Loddenkemper R. Thoracoscopy: state of the art. Eur Respir J 1998; 11: 213-21.

4. Lee P, Colt HG. State of the art: pleuroscopy. J Thorac Oncol 2007; 2: 663-70.

5. Ernst A, Silvestri GA, Johnstone D. Interventional Pulmonary Procedures: Guidelines from the American College of Chest Physicians. Chest 2003; 123: 16931717.

6. Astoul P, Seitz B, Boutin C. Diagnostic thoracoscopy in short-term hospitalisation. Acta Endosc 1990; 20: 79-83.

7. Boutin C, Viallat JR, Aelony Y. Practical Thoracoscopy. Berlin, Springer Verlag 1991.

8. Antony VB, Loddenkemper R, Astoul P, et al. Management of malignant pleural effusions. Am J Respir Crit Care Med 2000; 162: 1987-2001.
9. Antunes G, Neville E, Duffy J, Ali N. BTS guidelines for the management of malignant pleural effusions. Thorax 2003; 58 Suppl 2: ii29-ii38.

10. Laws D, Neville E, Duffy J, et al. BTS guidelines for the insertion of a chest drain. Thorax 2003; 58 (suppl II): ii53-ii59.

11. Luckraz H, Rammohan KS, Phillips M, et al. Is an intercostals chest drain necessary after video-assisted thoracoscopic (VATS) lung biopsy? Ann Thorac Surg 2007 Jul; 84: 237-9.

12. Blanc FX, Atassi K, Bignon J, Housset B. Diagnostic value of medical thoracoscopy in pleural disease: A 6year retrospective study. Chest 2002; 121: 1677-1683.

13. Izbicki G, Shitrit D, Yarmolovsky A, et al. Is routine chest radiography after transbronchial biopsy necessary?: A prospective study of 350 cases. Chest 2006; 129: 1561-4.

14. Garofalo G, Busso M, Perotto F, De Pascale A, Fava C. Ultrasound diagnosis of pneumothorax. Radiol Med (Torino) 2006; 111: 516-25.

15. Lichtenstein DA, Mezière G, Lascols N, et al. Ultrasound diagnosis of occult pneumothorax. Crit Care Med 2005; 33: 1425-6.

16. Reissig A, Kroegel C. Accuracy of transthoracic sonography in excluding post-interventional pneumothorax and hydropneumothorax. Comparison to chest radiography. Eur J Radiol 2005;53:463-70.

17. Dente CJ, Ustin J, Feliciano DV, et al. The accuracy of thoracic ultrasound for detection of pneumothorax is not sustained over time: a preliminary study. J Trauma 2007; 62: 1384-9.

18. Horsley A, Jones L, White J, Henry M. Efficacy and complications of small-bore, wire guided chest drains. Chest 2006; 130: 1857-1863.

19. Baumann MH. What size chest tube? What drainage system is ideal? And other chest tube management questions. Curr Opin Pulm Med 2003; 9: 276-81.

20. Lehwaldt D, Timmins F. The need for nurses to have in service education to provide the best care for clients with chest drains. J Nurs Manag 2007; 15: 142-8.

21. Lehwaldt D, Timmins F. Nurses' knowledge of chest drain care: an exploratory descriptive survey. Nurs Crit Care 2005; 10: 192-200.

22. Boutin C, Astoul P. Diagnostic thoracoscopy. Clin Chest Med 1998; 19: 295-309.

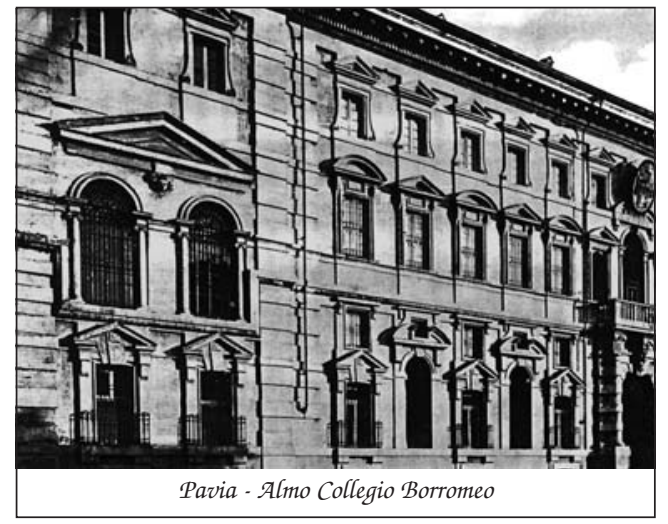

